\title{
Oxygen prescription and safe administration on respiratory wards
}

\author{
Authors: Christopher Dixon and Laura Jones
}

\section{Aims}

We aimed to assess staff knowledge of the safe use of oxygen devices and to audit oxygen prescribing and monitoring on the respiratory wards at a large teaching hospital, including the acute respiratory care and respiratory high-dependency units; to ensure that oxygen was used correctly as per British Thoracic Society (BTS) guidelines.

\section{Methods}

To assess practices, we audited against the gold standard of the 2008 British Thoracic Society (BTS) guidelines for emergency oxygen use in adult patients. Our hospital uses an inpatient drug chart that requires doctors to prescribe oxygen and target saturations. In cycle one (June 2015), we audited all inpatient drug and observation charts over 6 days to ensure that target saturations were prescribed and that patients had saturations measured. A questionnaire was distributed to test nursing staff's knowledge about the administration of oxygen and use of administration devices. After cycle one, we introduced a poster that educated staff and also sought to teach staff through ward education sessions. We adopted a multidisciplinary team approach to ensuring patient safety, presenting at clinical governance, and encouraging nurses and pharmacy to flag up to doctors when oxygen prescriptions were not filled in on the drug chart. After 4 months, we re-audited practice over 3 days and redistributed our questionnaire.

\section{Results}

In cycle one, $74 \%$ of 91 drug charts audited had no written prescription for oxygen; $16 \%$ of patients on oxygen had no prescription. Nursing staff regularly checked patient saturations but demonstrated poor knowledge of use of oxygen administration devices and the reasons why oxygen target saturations had to be prescribed. After staff education and help from our pharmacy colleagues, cycle two showed that only $5 \%$ of 47 charts audited of patients on oxygen had no prescription and $85 \%$ of all inpatients had a prescription. Staff knowledge of the safe use of oxygen devices was improved.

Authors: Bradford Royal Infirmary, Bradford, Yorkshire, UK

\section{Conclusions}

BTS audits of oxygen therapy have consistently shown oxygen prescription to be poor. In the 2013 BTS audit, 45\% of inpatients had no prescription for oxygen and $6.2 \%$ of patients were on oxygen therapy without a prescription. We have shown that staff education and making safe oxygen administration the responsibility of all staff can have a sustained effect on safety. Practices on the respiratory wards are now better than the perceived 'national standard' of oxygen prescribing as identified by the 2013 BTS audit.

\section{Conflict of interest statement}

None. 